P.J. Heikkinen - S. Autti - V.B.

Eltsov · J.J. Hosio • M. Krusius · V.V.

Zavjalov

\title{
Relaxation of Bose-Einstein Condensates of Magnons in Magneto-Textural Traps in Superfluid ${ }^{3} \mathrm{He}-\mathrm{B}$
}

Received: date / Accepted: date

\begin{abstract}
In superfluid ${ }^{3} \mathrm{He}-\mathrm{B}$ externally pumped quantized spin-wave excitations or magnons spontaneously form a Bose-Einstein condensate in a 3-dimensional trap created with the order-parameter texture and a shallow minimum in the polarizing field. The condensation is manifested by coherent precession of the magnetization with a common frequency in a large volume. The trap shape is controlled by the profile of the applied magnetic field and by the condensate itself via the spin-orbit interaction. The trapping potential can be experimentally determined with the spectroscopy of the magnon levels in the trap. We have measured the decay of the ground state condensates after switching off the pumping in the temperature range $(0.14 \div 0.2) T_{\mathrm{c}}$. Two contributions to the relaxation are identified: (1) spin diffusion with the diffusion coefficient proportional to the density of thermal quasiparticles and (2) the approximately temperature-independent radiation damping caused by the losses in the NMR pick-up circuit. The measured dependence of the relaxation on the shape of the trapping potential is in a good agreement with our calculations based on the magnetic field profile and the magnon-modified texture. Our values for the spin diffusion coefficient at low temperatures agree with the theoretical prediction and earlier measurements at temperatures above $0.5 T_{\mathrm{c}}$.
\end{abstract}

Keywords superfluid ${ }^{3} \mathrm{He} \cdot$ spin wave $\cdot$ magnons $\cdot$ BEC $\cdot$ coherent spin precession $\cdot$ spin diffusion $\cdot$ radiation damping

P.J. Heikkinen · S. Autti · V.B. Eltsov · J.J. Hosio · M. Krusius · V.V. Zavjalov O.V. Lounasmaa Laboratory, Aalto University, P.O. Box 15100, 00076 AALTO, Finland

E-mail: petri.heikkinen@aalto.fi 


\section{Introduction}

One of the interesting recent developments in condensed-matter physics is the extension of the concept of Bose-Einstein condensation to bosonic quasiparticles in solid-state and liquid systems [1, 2]. This concept is applicable to systems of pumped quasiparticles if the partial equilibrium within such a system with an approximately constant number of quasiparticles is reached faster than the full thermodynamic equilibrium, where the quasiparticle number is not conserved. By pumping, the quasiparticle number can be kept close to constant and the system stays in a dynamic steady state. Alternatively, one can study slowly decaying condensates after the pumping is stopped, which is similar to the case of a condensate of cold atoms in an optical trap where the particle number continuously decreases due to evaporation and recombination processes. Bose-Einstein condensation of quasiparticles has been demonstrated for magnons in various systems [3, 4, 5], for excitonpolaritons [6], and even for photons [7].

In superfluid ${ }^{3} \mathrm{He}-\mathrm{B}$ magnons are pumped using NMR techniques and their condensation is manifested by spontaneous long-lived coherent precession of the magnetization in an external magnetic field. At temperatures above $0.4 T_{\mathrm{c}}$ the condensate emerges as a so called homogeneously precessing domain (HPD) [8, 9]. In the HPD the uniformly precessing magnetization is deflected from the magnetic field by an angle exceeding $104^{\circ}$. The domain typically occupies a large fraction of the sample volume bounded by the walls of the container. If part of the sample is not involved in the precession, it is separated from the HPD by a relatively thin boundary where the tipping angle of magnetization rapidly changes. In the absence of pumping the HPD decays owing to the Leggett-Takagi relaxation originating from the coupling of spins of the normal and superfluid components, and owing to the spin diffusion through the boundary [10]. Additionally, at temperatures below about $0.4 T_{\mathrm{c}}$ the HPD experiences highly accelerated decay known as "catastrophic relaxation" owing to the Suhl type instability [1].

At even lower temperatures, below about $0.3 T_{c}$, another type of magnon $\mathrm{BEC}$, the so-called trapped condensate, is observed in ${ }^{3} \mathrm{He}-\mathrm{B}[12,3]$. In this case magnons are confined to a 3-dimensional trap formed by the combined effect of the spin-orbit interaction in a spatially inhomogeneous texture of the order-parameter orbital anisotropy axis and the minimum of the Zeeman energy in the applied magnetic field, Fig. 1. Such traps can be prepared within bulk ${ }^{3} \mathrm{He}-\mathrm{B}$ far from walls. The magnon density in such condensates can be much smaller than in the HPD resulting in tipping angles of the magnetization $\beta_{M} \sim 1^{\circ} \div 10^{\circ}$ or less. Also there is no sharp boundary of the condensate, but $\beta_{M}$ decreases gradually from the center of the trap as prescribed by an appropriate eigenstate wave function in the trap.

At temperatures where the trapped magnon condensates are observed, Leggett-Takagi relaxation becomes negligible and spin diffusion was identified as the main mechanism responsible for the temperature dependence of the relaxation of such condensates [13]. As the density of the normal component rapidly decreases with decreasing temperature, the lifetime of the condensates increases and can exceed half an hour in the case when the con- 
densate is well isolated from the walls [14]. Eventually, the lifetime is limited by an approximately temperature-independent relaxation process [13] which has not been identified before this work.

One of the characteristic features of the condensates in the magnetotextural trap comes from the fact that the order-parameter texture is not perfectly rigid and can be modified in the presence of the precessing spin owing to the spin-orbit interaction, i.e., magnons affect their trapping potential. This "self-trapping" property has many interesting consequences [15, 16]. In particular, it explains why the frequency of the precession in the condensate increases during relaxation. However, simultaneously this makes the analysis of the relaxation processes more complicated. In this report we present measurements on the relaxation of the magnon condensates at relatively low density, $\beta_{M} \lesssim 5^{\circ}$, where the back reaction on the texture is small. In this case the relaxation of the transverse magnetization $M_{\perp}$ is perfectly exponential $M_{\perp} \propto \exp \left(-t / \tau_{M}\right)$.

The temperature dependence of the relaxation time constant $\tau_{M}$ confirms the contributions from spin diffusion together with a temperatureindependent relaxation mechanism. With the spectroscopy of the ground and excited magnon levels in the trap we determine accurately the trapping potential and the corresponding condensate wave functions, which allows us to calculate values of the spin diffusion coefficient from the measured $\tau_{M}$. The results are in a good agreement with D. Einzel's theory 17] which earlier was favorably compared with measurements of spin diffusion at higher temperatures using HPD [10].

Our measurements on the relaxation as a function of the precession frequency show that the main source for the temperature-independent contribution to the relaxation is radiation damping [18]: The precessing magnetization of the condensate induces a current in the NMR pick-up coil which leads to dissipation in the active impedance of the measuring electric circuit. When the trapping potential is modified, the condensate spatial extent changes. This affects dissipation both due to spin diffusion and radiation damping. Our calculations of the combined effect are in a good agreement with the observed dependence of the relaxation on the profile of the applied magnetic field.

\section{Experimental setup}

The superfluid ${ }^{3} \mathrm{He}-\mathrm{B}$ sample fills a $15 \mathrm{~cm}$ long cylindrical container made from fused quartz, Fig. 11 The center of the magnet system used for these measurements is placed at $10 \mathrm{~mm}$ from the upper wall of the cylinder. The static magnetic field of about $26 \mathrm{mT}$ for the NMR measurements is oriented along the axis of the cylinder and has an inhomogeneity $\Delta H / H \approx 4 \cdot 10^{-4}$. An additional pinch coil creates the field in the opposite direction to that of the main solenoid and thus the total field has a minimum in the axial direction. The depth of the minimum can be controlled in the range $0-1 \mathrm{mT}$ by changing the current $I_{\min }$ in the pinch coil in the range $0-4 \mathrm{~A}$. The NMR pick-up coil has two sections wound from copper wire and is part of a tank 


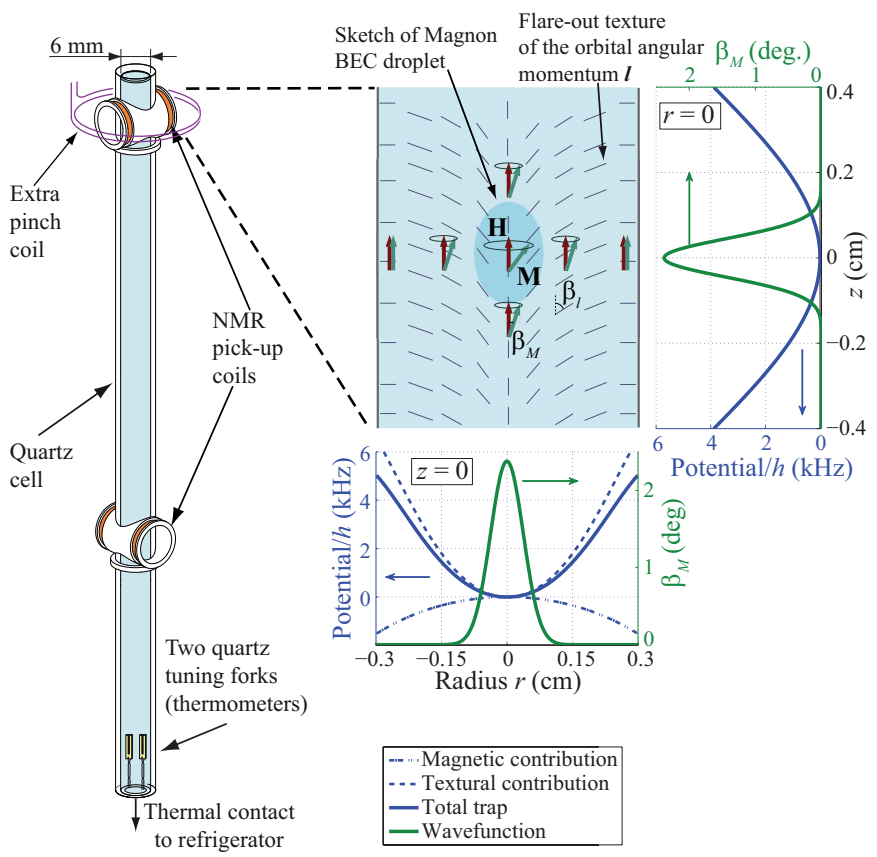

Fig. 1 (Color online) Sketch of the experimental setup and calculated trapping potentials for magnons. (Left) The sample container is equipped with two NMR spectrometers and tuning-fork thermometers. In the upper spectrometer, which is used in this work, a special pinch coil provides a minimum of the polarizing field in the axial direction. (Right) A sketch of the magnon condensate. In the condensate the magnetization $\mathbf{M}$ is precessing around the axially oriented magnetic field $\mathbf{H}$ with the same frequency and coherent phase despite magnetic field and textural inhomogeneities. The orientation of the orbital anisotropy axis $\hat{\mathbf{l}}$ is shown with short segmented lines. The two plots show the trapping potential for $P=0.5 \mathrm{bar}$, $T=0.14 T_{\mathrm{c}}$, and current $I_{\min }=2.0 \mathrm{~A}$ in the pinch coil, calculated respectively at $r=0$ or at $z=0$ (blue lines). The trap is a combination of the axial minimum in the Zeeman energy and the interaction energy between the precessing spin and the orbital texture which provides trapping in the radial direction. The profile of the ground-state wave function in this potential is shown with green lines.

circuit tuned to $826 \mathrm{kHz}$ frequency with a $\mathrm{Q}$ value of about 130 . The same coil is used to pump magnons.

Temperature is measured using a quartz tuning fork thermometer 19 installed inside the sample tube approximately $20 \mathrm{~mm}$ above its lower end, which opens to the liquid ${ }^{3} \mathrm{He}$ volume with the sintered heat exchanger on the nuclear cooling stage. As the scattering of thermal quasiparticles from the sample walls is probably diffusive and there is a heat leak to the sample of the order of $12 \mathrm{pW}$ [20], some temperature difference is expected along the cylinder from the NMR spectrometer to the thermometer fork. However, this possible temperature difference is small and does not affect the interpretation of results, as discussed below.

The magnetic part of the trapping potential is due to the Zeeman energy $F_{\mathrm{Z}}=\hbar \omega_{\mathrm{L}}(r, z)|\Psi|^{2}$, where $\omega_{\mathrm{L}}(r, z)=\gamma H(r, z)$ is the local Larmor frequency 
in the magnetic field $H(r, z), \gamma$ is the absolute value of the gyromagnetic ratio, $\Psi$ is the wave function of the magnon condensate, and the magnon density is $|\Psi|^{2}=\left(S-S_{z}\right) / \hbar=(\chi H / \gamma \hbar)\left(1-\cos \beta_{M}\right)$. Here $S=\chi H / \gamma$ is the equilibrium spin density, $\chi$ is the magnetic susceptibility of the B phase, and the magnon density is characterized in terms of the tipping angle of magnetization $\beta_{M}: S_{z}=S \cos \beta_{M}$. As shown by the profiles of the potential in Fig. 1. our coil system produces an approximately quadratic minimum of the Zeeman energy in the axial direction and an approximately quadratic maximum in the radial direction.

The textural part of the trapping potential comes from the spin-orbit interaction energy [2]

$$
F_{\text {so }}=\frac{4 \Omega_{\mathrm{B}}^{2}}{5 \omega_{\mathrm{L}}} \sin ^{2} \frac{\beta_{l}(r, z)}{2}|\Psi|^{2}
$$

Here we omitted terms quadratic in magnon density. The strength of the spinorbit interaction is characterized by the Leggett frequency $\Omega_{\mathrm{B}}$. The spatial dependence comes from the variation of the angle $\beta_{l}$ between the orbital anisotropy axis $\hat{\mathbf{l}}$ and the magnetic field. In a cylindrical sample in the axial magnetic field the texture of $\hat{\mathbf{l}}$ usually takes an axially-symmetric flare-out form, so that $\beta_{l}=0$ at the axis and grows to $\beta_{l}=\pi / 2$ at the cylindrical wall. According to Eq. (1) this results in a minimum of the spin-orbit energy in the radial direction, which is approximately quadratic at the bottom, as $\beta_{l}$ close to the axis changes linearly with distance $r$ from the axis.

The total confinement potential is the sum of the Zeeman and spin-orbit interaction energies. Close to the center of the trap it turns out to be nearly harmonic, and thus the spectrum of standing spin waves becomes the familiar spectrum of eigenstates in an axially symmetric harmonic trap 21

$$
\omega_{n_{\phi} n_{r} n_{z}}=\omega_{\mathrm{L}}(r=0, z=0)+\omega_{r}\left(\left|n_{\phi}\right|+n_{r}+1\right)+\omega_{z}\left(n_{z}+1 / 2\right),
$$

where $n_{\phi}, n_{r}$ and $n_{z}$ are azimuthal, radial and axial quantum numbers, respectively. Their allowed values are:

$$
n_{\phi}=0, \pm 1, \pm 2, \ldots, \quad n_{r}=0,2,4,6, \ldots, \quad n_{z}=0,1,2, \ldots .
$$

In Eq. (2) $\omega_{r}$ and $\omega_{z}$ are the radial and axial oscillator (or trapping) frequencies, respectively. By measuring the current induced in the NMR pickup coil we actually measure the transverse magnetization of the sample, $M_{\perp}=\chi H \int \sin \beta_{M}(r, z) d V$. Thus we can observe states only with the azimuthal number $n_{\phi}=0$ because of the antisymmetricity of the magnon wave function and $\beta_{M}$ for other values of $n_{\phi}$. For the same reason only states with even axial number $n_{z}$ are seen. Thus the spectrum of the lowest-lying magnon states in our trap is

$$
\omega_{n_{r} n_{z}}=\omega_{\mathrm{L}}(r=0, z=0)+\omega_{r}\left(n_{r}+1\right)+\omega_{z}\left(n_{z}+1 / 2\right)
$$

with even $n_{r}$ and $n_{z}$. Note that for these symmetric states $M_{\perp} \propto \int|\Psi| d V \propto$ $\sqrt{N_{\mathrm{m}} V_{\mathrm{m}}}$, where $N_{\mathrm{m}}$ is the number of magnons in the trap and $V_{\mathrm{m}}$ is the volume occupied by the condensate. 


\section{Spectroscopy of the magnon levels in the trap}

Pumping of magnons with continuous-wave (cw) or pulsed NMR techniques is usually coherent, since the frequency of rf pumping, $\omega_{\mathrm{rf}}$, fixes the magnon chemical potential, and the orientation of the rf field sets the phase of the precession of the magnetization. As the magnons in the trap relax towards the ground state (and lower precession frequencies), this coherence is lost, though, and the ground-state condensate develops spontaneously coherent precession at the frequency $\omega_{00}<\omega_{\text {rf }}$ with the phase of its precession unrelated to that of the rf excitation field [22, 16]. This remarkable feature of Bose-Einstein condensation has also been demonstrated using incoherent pumping with white noise [23].

Thus, observation of the ground-state magnon BEC is reasonably straightforward when a proper 3-dimensional trap is created and the temperature is sufficiently low to increase the lifetime of the condensate. Pumping then has to be applied at a frequency above $\omega_{00}$ either as a spectrally wide pulse or, if using narrow-band cw-NMR excitation, it has to coincide with one of the excited levels $\omega_{n_{r} n_{z}}$ in the trap. The response is then measured at $\omega_{00}$ frequency. The off-resonant excitation is practical since it allows to separate the frequencies of excitation and measurement and to use the same NMR coil for both purposes without interference.

To determine the frequencies $\omega_{n_{r} n_{z}}$ of all excited levels accurately, a special approach should be used. We rely on the experimental observation (which remains so far unexplained) that for the off-resonant excitation to be effective some excitation threshold should be overcome. In the experiment we decrease the amplitude of rf pumping until we notice that magnons pumped to the excited levels do not relax to the ground level. Then the frequency $\omega_{\text {rf }}$ of the cw pumping can be swept to determine the positions of all levels. An example of such a measurement is presented in Fig. 2. In the main panel the amplitude of the Fourier spectrum of the signal from the NMR coil is shown as a function of time. The diagonal line represents the frequency sweep of the NMR excitation. When it crosses the frequencies of the magnon levels, the corresponding precessing signals are excited as shown by the decaying vertical traces. In this example, the precession frequencies of the condensates do not change during the decay since the magnon density is so small from the start that the self-modification of the textural potential is negligible.

The magnons on the excited levels of the trap demonstrate spontaneously coherent precession, characteristic for the BEC: The precession frequency during the decay is not related to the rf pumping frequency, which is continuously changing, and the decay time of several seconds significantly exceeds the decoherence time of linear NMR, which in our conditions is less than $3 \mathrm{~ms}$. This is another demonstration of the excited-level BEC discussed in Ref. [16].

More standard cw-NMR spectra of standing spin waves can be measured with the detection locked to the excitation frequency, see inset in Fig. 2 , A small enough pumping amplitude and a sweep towards higher frequencies, unlike in Refs. 16, 24], are used to avoid off-resonant excitation and a large non-linear signal due to the phenomenon of self-trapping. Thus the frequen- 


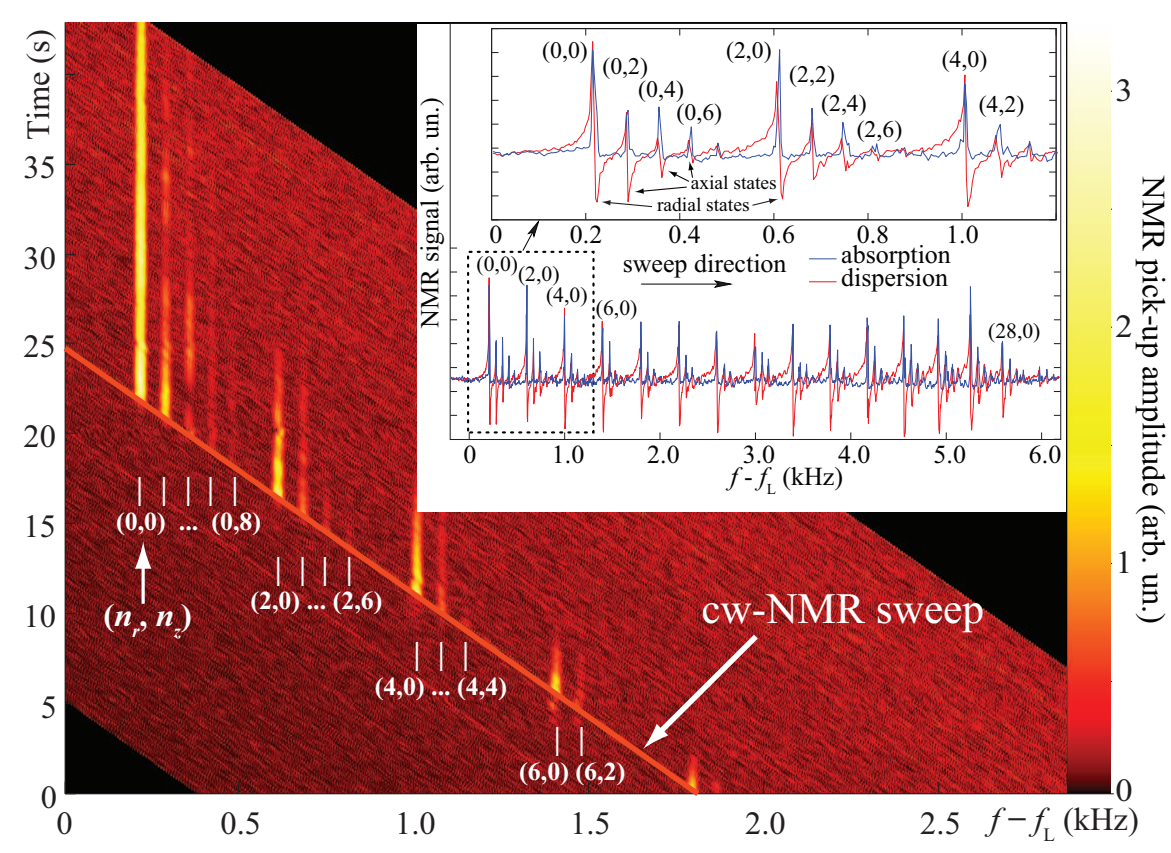

Fig. 2 (Color online) Measurement of the magnon levels in the trapping potential using cw-NMR sweep at a very low excitation amplitude. The main plot shows the color-coded amplitude of the Fourier spectrum of the signal from the NMR pick-up coil as a function of time. The time window of the Fourier transform is $0.8 \mathrm{~s}$. The vertical white lines mark the magnon levels $\left(n_{r}, n_{z}\right)$ in the harmonic approximation, Eq. (4), with $\omega_{r} / 2 \pi=197 \mathrm{~Hz}, \omega_{z} / 2 \pi=35 \mathrm{~Hz}$ and $f_{\mathrm{L}}=\omega_{\mathrm{L}} / 2 \pi=826 \mathrm{kHz}$. The vertical measured traces emerging at these frequencies represent coherent precession of the magnon condensates at the corresponding trap levels. The longest trace belongs to the ground state $n_{r}=n_{z}=0$. The inset shows the traditional cw-NMR spectrum measured at the same conditions as in the main panel: $P=0.5 \mathrm{bar}$, $T=0.14 T_{\mathrm{c}}$, and $I_{\min }=0.25 \mathrm{~A}$. Absorption peaks are seen at the frequencies where magnons are injected to the trap levels with quantum numbers marked near the peaks.

cies of the maxima in the absorption signal provide a good measure of the magnon levels $\omega_{n_{r} n_{z}}$. All the radial states up to the limit put by the Leggett frequency $\Omega_{\mathrm{B}}$ in Eq. (1) can be observed. It should be noted that such traditional cw-NMR measurements have limited use in the studies of the trapped magnon condensates since they cannot provide information about the offresonant excitation or the long lifetime of the different condensate states.

A few of the lowest magnon energy levels are plotted as a function of the current in the minimum coil $I_{\min }$ in Fig. 3. These follow reasonably well the harmonic approximation Eq. (4) with $\omega_{z} \propto \sqrt{I_{\min }}$ and $\omega_{r}$ decreasing approximately linearly with $I_{\min }$. These dependences are expected for the axial minimum of the Zeeman energy with the depth proportional to $I_{\min }$ and the radial maximum of the Zeeman energy which adds to a deeper radial minimum of the textural energy, see Fig. 11 The textural energy itself does not change substantially due to the small change of the static field provided by the 


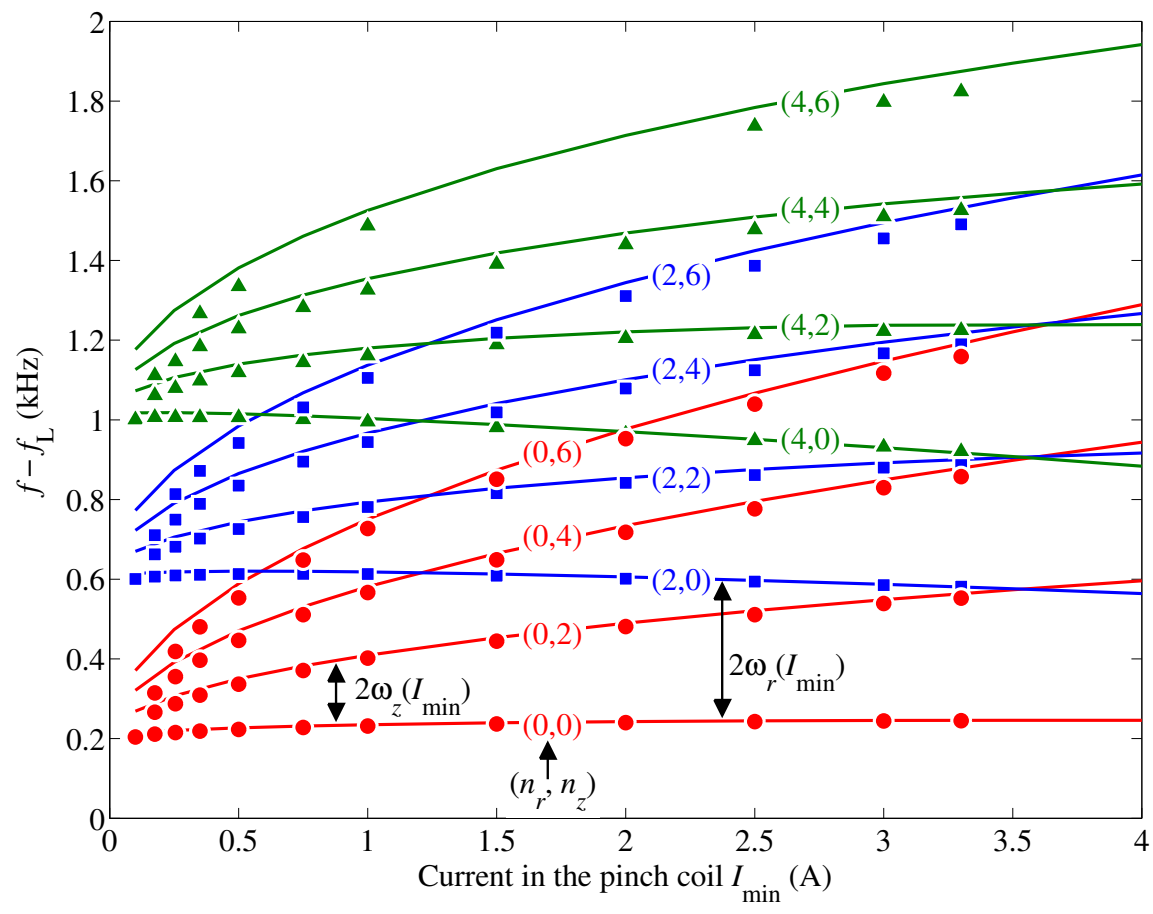

Fig. 3 (Color online) Frequencies of the lowest-lying energy levels in the magnon trap as a function of the current $I_{\min }$ in the coil creating the minimum in the polarizing field. Measurements (symbols) are compared to calculations (lines) of the eigenstates of the model trapping potential. The measurements are performed at $T=0.15 T_{\mathrm{c}}$ but in practice the trapping potential is temperature-independent below $0.2 T_{\mathrm{c}}$.

pinch coil. In most of the experimental range $\omega_{z}<\omega_{r}$, i.e., the condensate droplet is elongated in the axial direction. As the quantum numbers get larger, the harmonic approximation breaks, because such states probe the texture with larger $\beta_{l}$ closer to the wall where $\sin \beta_{l}$ is no more proportional to $r$, and because of the anharmonicity of the field profile at larger distances from the center of the trap.

In Fig. 3 we compare the measured level frequencies with the calculations beyond the harmonic approximation. The solid lines represent the solution of the eigenvalue problem in the realistic model of the $3 \mathrm{D}$ trapping potential. In this model we calculate the textural part of the potential using the methods of Refs. [25, 26] without fitting parameters. The magnetic part of the potential is calculated from the geometry of the solenoidal superconducting coils with an extra fitting parameter for the screening of the magnetic field by the superconducting parts of the setup. The agreement between calculations and experiment in Fig. 3 is reasonably good, and the same model of the magnetic field properly describes also the shape of the NMR response in normal ${ }^{3} \mathrm{He}$. In the next section we use the shape of the ground-state wave function in the 
model potential to analyze quantitatively the measurements of the relaxation of the ground-state condensate.

\section{Relaxation measurements}

After the magnon condensate is created with cw or pulsed NMR and pumping is turned off, the condensate decays. When the off-resonant excitation with sufficiently large pumping amplitude is used, then, unlike in Fig. 2, magnons from the excited levels in the trap quickly relax to the ground state, which becomes the only precessing signal living for a long time. We measure the frequency $f_{\mathrm{m}}(t)=\omega_{00} / 2 \pi$ and amplitude $A_{\mathrm{m}}(t)$ of the signal in the pick-up coil induced by the precessing magnetization of the decaying condensate using Fourier transformation. An example of such a measurement is shown in Fig. 4 a. The final part of the decay is nicely exponential: $\left.f_{\mathrm{m}}\right|_{t} ^{\infty} \propto \exp \left(-t / \tau_{\text {freq }}\right)$ and $A_{\mathrm{m}}(t) \propto \exp \left(-t / \tau_{M}\right)$. The frequency $f_{\mathrm{m}}=\left(\omega_{\mathrm{L}}+\omega_{r}\left(N_{\mathrm{m}}(t)\right)+\omega_{z} / 2\right) /(2 \pi)$ increases while the number of magnons in the trap $N_{\mathrm{m}}$ decreases as a result of the modification of the textural trapping potential by magnons which leads to $d \omega_{r} / d N_{\mathrm{m}}<0$ [16]. The amplitude $A_{\mathrm{m}}$ depends both on the transverse magnetization of the condensate $M_{\perp}$ and its geometrical shape. However, in the final part of the decay when the textural potential has its final form $A_{\mathrm{m}} \propto M_{\perp} \propto \sqrt{N_{\mathrm{m}}}$. For small $N_{\mathrm{m}}$, roughly $\left.f_{\mathrm{m}}\left(N_{\mathrm{m}}\right)\right|_{N_{\mathrm{m}}} ^{0} \propto M_{\perp}^{2}\left(N_{\mathrm{m}}\right)$ [27], and this explains why in the measurements $\tau_{\text {freq }}$ is roughly half of $\tau_{M}$.

The measured temperature dependence of the relaxation rate $1 / \tau_{M}$ is plotted in Fig. 4b. The horizontal axis shows the width of the resonance of the thermometer fork. This is to emphasize that the relaxation rate is an approximately linear function of the density of thermal quasiparticles. A similar dependence was observed earlier in Ref. [13], where it was connected to the temperature dependence of the spin diffusion relaxation mechanism. The spin diffusion coefficient quickly decreases with temperature at low temperatures owing to the Leggett-Rice effect [28]. Fig. 40 also demonstrates that the extrapolation of relaxation to zero temperature (i.e. close to zero fork width) results in a finite value. This is also consistent with Ref. 13] where an approximately temperature-independent relaxation mechanism was found, in addition to spin diffusion. With a good knowledge of the magnon condensate density profile we can extract values for the diffusion coefficient from the measurements of the relaxation rate. Before that, however, we proceed to the identification of the temperature-independent relaxation mechanism.

The main assumption in the following considerations is that the decay of the condensate is relatively slow, so that the superfluid spin currents within the condensate always support it in a quasi-equilibrium state. That is, for a given number of magnons $N_{\mathrm{m}}$ in the condensate we calculate its wave function and self-consistent trapping potential regarding the condensate as stationary, as in Ref. 27]. This results in well-defined dependences for the total energy of the condensate $E\left(N_{\mathrm{m}}\right)$ and its transverse magnetization $M_{\perp}\left(N_{\mathrm{m}}\right)$. Additionally, since the spin-orbit interaction energy is about four orders of magnitude smaller than the Zeeman energy in our conditions, we assume that $E=\int F_{\mathrm{Z}} d V$. 


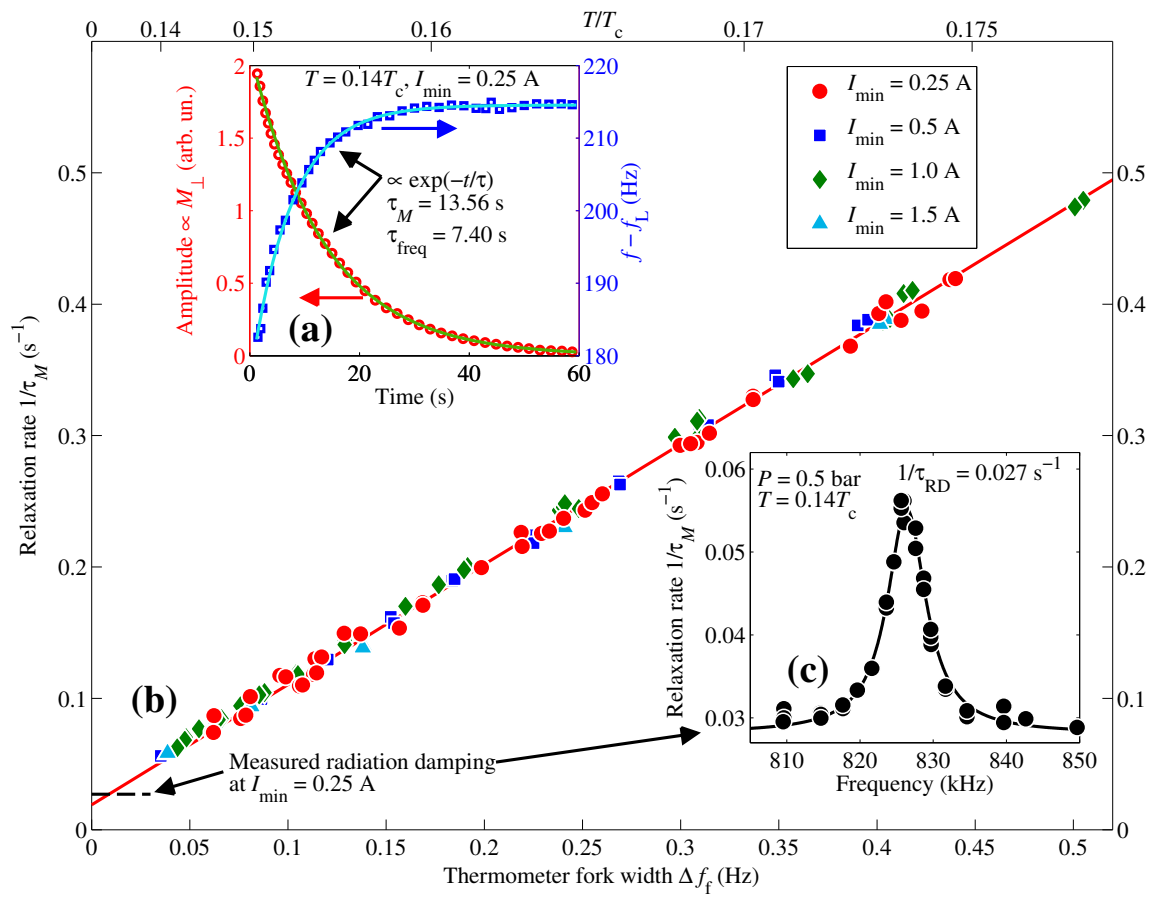

Fig. 4 (Color online) Relaxation rate of the magnon BEC. (a) An example of the relaxation measurement where the amplitude (circles) and the frequency (squares) of the precessing signal are fit with the exponential decay curves (lines). (b) Dependence of the relaxation rate $1 / \tau_{M}$ of the magnetization on the resonance width of the thermometer quartz tuning fork (symbols) demonstrates nearly linear dependence (solid line) on the density of thermal quasiparticles $\left[\propto \exp \left(-\Delta / k_{\mathrm{B}} T\right)\right]$ to which the fork is sensitive. Such temperature dependence is explained by spin diffusion, while the extrapolated finite zero-temperature relaxation is caused by radiation damping. (c) Dependence of the relaxation rate on the precession frequency (circles) shows the effect of the $T$-independent radiation damping at $I_{\min }=0.25 \mathrm{~A}$. The solid line is a fit to the square of the Lorentzian response curve of the NMR pick-up resonance circuit, where the height of the peak determines the value of the radiation damping and the offset in the wings is due to the spin diffusion at the temperature of the measurement.

Radiation damping. A measurement of the relaxation rate as a function of the precession frequency $\omega_{00}$ reveals a dependence similar to the resonant response of the NMR tank circuit, Fig. 4. Here the frequency $\omega_{00}$ is controlled by changing $\omega_{\mathrm{L}}$ with the external magnetic field. Such frequency dependence is explained by the relaxation mechanism known as the radiation damping [18]

$$
d E / d t=-V_{\mathrm{s}}^{2} /(2 R)
$$

where $V_{\mathrm{s}}$ is the induced voltage on the pick-up coil due to the precessing magnetization and $R$ is the active impedance of the resonance circuit. Expressing $E$ via $M_{\perp}$ as noted above we get from Eq. (5) the relaxation of 
magnetization with the rate

$$
\tau_{\mathrm{RD}}^{-1}=\frac{V_{\mathrm{S}}^{2}}{2 R M_{\perp}} \frac{d M_{\perp}}{d E} .
$$

Since $V_{\mathrm{s}} \propto M_{\perp}$ and $E \approx \hbar \omega_{\mathrm{L}} N_{\mathrm{m}} \propto M_{\perp}^{2}$ the rate is approximately $M_{\perp}$ independent, i.e., the relaxation is exponential. It should be noted that the radiation damping increases with the increase of the quality factor $Q \propto 1 / R$ of the tank circuit. The magnetic flux through the pick-up coil, and thus the induced voltage $V_{\mathrm{s}}$, depends on the profile of the magnon density in the condensate, but since the trapping potential is almost $T$-independent in the studied temperature range the radiation damping is also temperatureindependent.

The frequency dependence of $V_{\mathrm{s}}$ induced in the tank circuit by a given alternating flux through the coil follows the standard Lorentzian response. Experimentally the radiation damping can be determined as a difference in relaxation rate between the maximum and the wings in Fig. 4. This value is plotted in Fig. 4b by the horizontal dashed line. As one can see, the radiation damping explains an essential part of the zero-temperature damping of the magnon BEC. The conclusion whether actually all the damping is explained in this way depends on the precise knowledge of the zero-temperature (or intrinsic) width of the thermometer fork, $\Delta f_{\mathrm{f}}^{\mathrm{i}}$. Unfortunately, this value is not known with sufficient precision, and thus is not taken into account in Fig. 4. On the two cool-downs of the cryostat during the course of these measurements the width of the fork resonance in vacuum was measured as 12 and $17 \mathrm{mHz}$ at $T=12-13 \mathrm{mK}$, while the extrapolation of the $1 / \tau_{M}$ temperature dependence in Fig. $4 \mathrm{~b}$ reaches $1 / \tau_{\mathrm{RD}}$ value at the fork width of $(9 \pm 4) \mathrm{mHz}$. This is consistent with the measured value $\Delta f_{\mathrm{f}}^{\mathrm{i}}=12 \mathrm{mHz}$ thus leaving no indications for additional damping mechanisms at zero temperature. If $\Delta f_{\mathrm{f}}^{\mathrm{i}}=17 \mathrm{mHz}$, then the unexplained zero-temperature damping of the magnon BEC would amount to about $25 \%$ of the observed radiation damping.

We might also note that no saturation is seen in the temperature dependence of relaxation at the lowest temperatures in Fig. 4 $\mathrm{b}$. This means that the temperature gradient along the sample column is sufficiently small and does not result in effects larger than the scatter in the measurements. The residual temperature difference between the thermometer and the NMR volume can be considered as a contribution to the uncertainty in $\Delta f_{\mathrm{f}}^{\mathrm{i}}$.

The spin diffusion contribution to the relaxation of the condensate is expressed as [29, 30]

$$
\frac{d E}{d t}=-\frac{D \gamma^{2}}{\chi}\left\langle\frac{\partial S_{i}}{\partial x_{j}} \frac{\partial S_{i}}{\partial x_{j}}\right\rangle,
$$

where $D$ is the transverse component of the spin-diffusion tensor and indices $i$ and $j$ correspond to spin components in the cartesian coordinates. The brackets denote averaging over the volume. The spin components can be expressed via the condensate wave function, and assuming its phase to be 

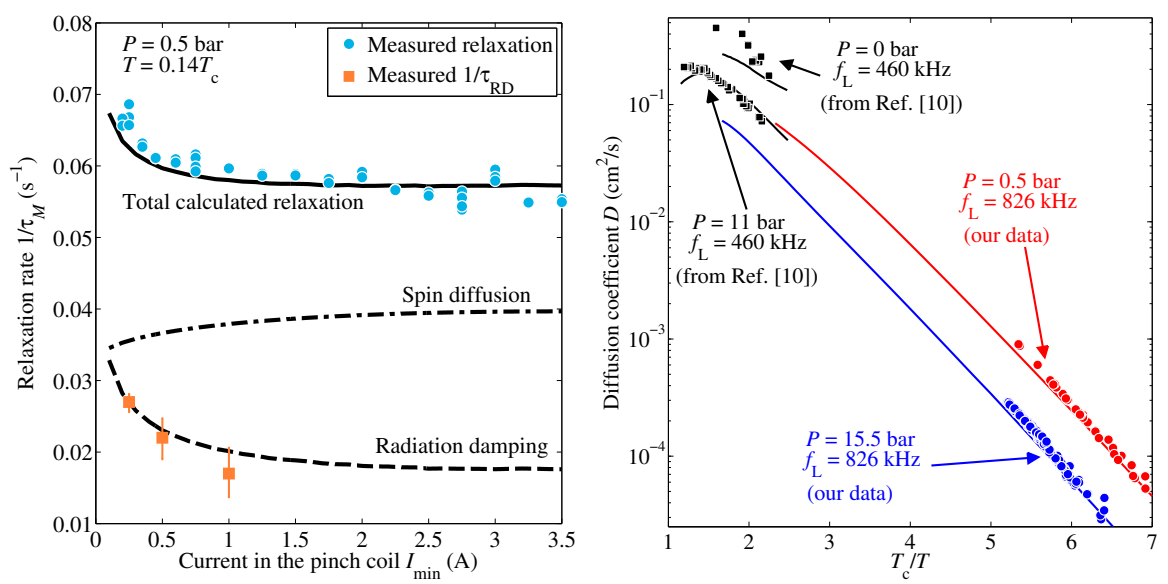

Fig. 5 (Color online) (Left) Relaxation rate of the magnon condensate as a function of the current $I_{\min }$ in the field minimum coil (circles). Independent measurements of the radiation damping executed in similar way as in Fig. 4k are shown with squares. The lines show the calculated dependencies for the radiation damping (scaled vertically to match the experimental values), for the spin diffusion with the diffusion coefficient taken from the right panel, and the total relaxation. (Right) The temperature dependence of the spin diffusion coefficient. Values extracted from the measurements in Fig. 4 at $I_{\min }=0.25 \mathrm{~A}$ (red circles) and from similar measurements at $P=15.5$ bar and $I_{\min }=2 \mathrm{~A}$ (blue circles) are compared to the theoretical prediction [17] (lines) and to the measurements at higher temperatures [10] (squares). Note that the measurements are done at different frequencies while, according to the theory, $D$ is approximately inversely proportional to $f_{\mathrm{L}}$ [17].

spatially uniform we arrive to the expression for the relaxation rate of the magnetization

$$
\tau_{\mathrm{SD}}^{-1}=\frac{\omega_{\mathrm{L}}^{2} \chi}{\gamma^{2}} \frac{D}{M_{\perp}} \frac{d M_{\perp}}{d E}\left[\int\left(\nabla \beta_{M}\right)^{2} d V\right] .
$$

We use this expression to fit the relaxation data in Fig. 40 using $D$ as a fitting parameter. We account only for the spin-diffusion and radiation-damping contributions to the relaxation, i.e., express $\tau_{M}^{-1}=\tau_{\mathrm{SD}}^{-1}+\tau_{\mathrm{RD}}^{-1}$, and thus selfconsistently take $\Delta f_{\mathrm{f}}^{\mathrm{i}}=9 \mathrm{mHz}$ in the temperature calibration. In calculating $\beta_{M}(r, z)$ and $d E / d M_{\perp}$ in Eq. (8) we use self-consistent calculations of the textural trapping potential and the wave functions. The texture modification by the condensate is relatively small, and the calculated relaxation rate is approximately independent of $M_{\perp}$. This is in agreement with the experiments.

The results for the temperature dependence of the diffusion coefficient are shown in the right panel of Fig. 5. The expected nearly-exponential decrease of $D$ at the lowest temperatures is observed. The measurements are compared to the theoretical prediction from Eq. (108) in Ref. 17]. In evaluating this expression we take the Fermi liquid parameters from Ref. [31], and we use the weak-coupling plus gap [32]. As in Ref. [17], we approximate the order-parameter $\hat{\mathbf{n}}$ vector to be oriented uniformly along the field direction. In principle this is not strictly valid for the trapped magnon BEC, but in 
Eq. (7) the main contribution comes from the condensate region where the deflection angle of $\hat{\mathbf{n}}$ from the magnetic field is below $15^{\circ}$, so we consider this assumption to be reasonable. Overall, we consider the agreement between the measurements and the theoretical value of $D$ as good, given that the theoretical expression has no fitting parameters.

Dependence of relaxation on the trapping potential. Both the spin diffusion and the radiation damping depend on the magnon condensate wave function. When the axial confinement of the condensate increases with increasing $I_{\min }$, spin diffusion increases due to the increase in the gradients of the spin. Simultaneously, radiation damping decreases due to the reductions in the spatial extent of the condensate and the effective filling factor for the NMR pick-up. The combined effect is measured in the left panel of Fig. 5, It is in a reasonable agreement with our calculations of the respective changes in the damping.

\section{Conclusions}

We have studied the relaxation of trapped Bose-Einstein condensates of magnons in superfluid ${ }^{3} \mathrm{He}-\mathrm{B}$ at temperatures below $0.2 T_{\mathrm{c}}$. For the first time the temperature-independent relaxation mechanism from radiation damping is identified. The measurements of the relaxation from spin diffusion allow us to determine reliably the spin diffusion coefficient $D$ at the lowest temperatures. The measured values of $D$ agree well with the theoretical prediction and the earlier measurements at higher temperatures. We have demonstrated the dependence of the relaxation on the shape of the trapping potential which is important for a proper analysis of the relaxation in the regime of large magnon density, where the self-modification of the trapping potential results in non-exponential effects. The achieved understanding of the relaxation of the magnon condensates in bulk ${ }^{3} \mathrm{He}-\mathrm{B}$ provides a firm basis for studying relaxation effects originating from exotic fermion bound states at the surfaces of ${ }^{3} \mathrm{He}-\mathrm{B}$ [33] or in the cores of quantized vortices [34].

Acknowledgements We thank Yu.M. Bunkov, V.V. Dmitriev, P. Hunger, P. Skyba and G.E. Volovik for useful discussions. This work has been supported in part by the EU 7th Framework Programme (FP7/2007-2013, Grant No. 228464 Microkelvin) and by the Academy of Finland through its LTQ CoE grant (project no. 250280). P.J.H. and J.J.H. acknowledge financial support from the Väisälä Foundation of the Finnish Academy of Science and Letters. 


\section{References}

1. D. Snoke, Nature 443, 403 (2006)

2. Yu.M. Bunkov, G.E. Volovik, in Novel Superfluids, volume 1, ed. by K.H. Bennemann, J.B. Ketterson (Oxford University Press, 2013), pp. 253-311; arXiv:1003.4889

3. Yu.M. Bunkov, G.E. Volovik, Phys. Rev. Lett. 98, 265302 (2007)

4. S.O. Demokritov, V.E. Demidov, O. Dzyapko, G.A. Melkov, A.A. Serga, B. Hillebrands, A.N. Slavin, Nature 443, 430 (2006)

5. Yu.M. Bunkov, E.M. Alakshin, R.R. Gazizulin, A.V. Klochkov, V.V. Kuzmin, V.S. L'vov, M.S. Tagirov, Phys. Rev. Lett. 108, 177002 (2012)

6. J. Kasprzak, M. Richard, S. Kundermann, A. Baas, P. Jeambrun, J.M.J. Keeling, F.M. Marchetti, M.H. Szymaska, R. André, J.L. Staehli, V. Savona, P.B. Littlewood, B. Deveaud, Le Si Dang, Nature 443, 409 (2006)

7. J. Klaers, J. Schmitt, F. Vewinger, M. Weitz, Nature 468, 545 (2010)

8. A.S. Borovik-Romanov, Yu.M. Bunkov, V.V. Dmitriev, Yu.M. Mukharskii, JETP Lett. 40, 1033 (1984)

9. I.A. Fomin, JETP Lett. 40, 1037 (1984)

10. Yu.M. Bunkov, V.V. Dmitriev, A.V. Markelov, Yu.M. Mukharskii, D. Einzel, Phys. Rev. Lett. 65, 867 (1990)

11. Yu.M. Bunkov, V.V. Dmitriev, Yu.M. Mukharskiy, J. Nyeki, D.A. Sergatskov, Europhys. Lett. 8, 645 (1989)

12. Yu.M. Bunkov, S.N. Fisher, A.M. Guénault, G.R. Pickett, Phys. Rev. Lett. 69, 3092 (1992)

13. S.N. Fisher, G.R. Pickett, P. Skyba, N. Suramlishvili, Phys. Rev. B 86, 024506 (2012)

14. S.N. Fisher, A.M. Guénault, A.J. Hale, G.R. Pickett, P.A. Reeves, G. Tvalashvili, J. Low Temp. Phys. 121, 303 (2000)

15. Yu.M. Bunkov, J. Low Temp. Phys. 138, 753 (2005)

16. S. Autti, Yu.M. Bunkov, V.B. Eltsov, P.J. Heikkinen, J.J. Hosio, P. Hunger, M. Krusius, G.E. Volovik, Phys. Rev. Lett. 108, 145303 (2012)

17. D. Einzel, J. Low Temp. Phys. 84, 321 (1991)

18. N. Bloembergen, V. Pound, Phys. Rev. 95, 8 (1954)

19. R. Blaauwgeers, M. Blazkova, M. Ĉloveĉko, V.B. Eltsov, R. de Graaf, J. Hosio, M. Krusius, D. Schmoranzer, W. Schoepe, L. Skrbek, P. Skyba, R.E. Solntsev, D.E. Zmeev, J. Low Temp. Phys. 146, 537 (2007)

20. J.J. Hosio, V.B. Eltsov, R. de Graaf, M. Krusius, J. Mäkinen, D. Schmoranzer, Phys. Rev. B 84, 224501 (2011)

21. L. Pauling, E.B. Wilson, Introduction to Quantum Mechanics (McGrawHill Book Company, Inc., 1935), p. 110

22. D.J. Cousins, S.N. Fisher, A.I. Gregory, G.R. Pickett, N.S. Shaw, Phys. Rev. Lett. 82, 4484 (1999)

23. S.N. Fisher, A.M. Guénault, G.R. Pickett, P. Skyba, Physica B 329-333, $80(2003)$

24. V.B. Eltsov, R. de Graaf, M. Krusius, D.E. Zmeev, J. Low Temp. Phys. 162, 212 (2011) 
25. E.V. Thuneberg, J. Low Temp. Phys. 122, 657 (2001)

26. J. Kopu, J. Low Temp. Phys. 146, 47 (2007)

27. S. Autti, V.B. Eltsov, G.E. Volovik, JETP Letters 95, 544 (2012)

28. A.J. Leggett, M.J. Rice, Phys. Rev. Lett. 20, 586 (1968)

29. I.A. Fomin, JETP Lett. 30, 164 (1979)

30. A.V. Markelov, Yu.M. Mukharsky, Physica B 178, 202 (1992)

31. E.R. Dobbs, Helium Three (Oxford University Press, 2000), p. 52

32. D. Rainer, J.W. Serene, Phys. Rev. B 13, 4745 (1976)

33. Suk Bum Chung, Shou-Cheng Zhang, Phys. Rev. Lett. 103, 235301 (2009)

34. N.B. Kopnin, G.E. Volovik, Phys. Rev. B 57, 8526 (1998) 